\title{
Wireless communication for firefighters using dual-polarized textile antennas integrated in their garment
}

\author{
Luigi Vallozzi, Patrick Van Torre, Carla Hertleer, Hendrik Rogier, Senior member IEEE, \\ Marc Moeneclaey, Fellow IEEE and Jo Verhaevert
}

\begin{abstract}
A compact wearable antenna system, completely made out of textile materials for integration into protective garments, is proposed. The system implements combined pattern and polarization diversity to improve the quality of the communication link. The performance of the on-body antenna system, integrated into a firefighter jacket worn by a test person, was investigated in an indoor measurement campaign. Several receiver diversity schemes and different combining techniques were evaluated in terms of bit error rate, signal-to-noise ratio and signal correlations. By comparing them to theoretical results, we demonstrate the reliability of the proposed system and the advantage of using diversity.
\end{abstract}

Index Terms-Body-centric communications, firefighter, pattern-polarization diversity, textile antenna, wearable antenna system.

\section{INTRODUCTION}

W EARABLE textile systems for use in body-centric communication create an interface between the wearer and the external world, by continuously monitoring the vital and activity functions of the body (i.e. temperature, heart rate, pressure), as well as the surrounding environment (i.e. humidity, temperature, etc.). On the one hand, once the data of the sensors integrated into the system are acquired, they need to be transmitted in a wireless way to a fixed base station. On the other hand, the fixed base station needs to send back data to the wearable system, for example alarms or other useful information.

A wearable textile system can be used in applications such as health monitoring of patients [1], coordination of interventions by rescue workers during emergencies [2], sports, entertainment and so on. In this article the particular application of data communication between a firefighter and a base station in an indoor environment is considered. More specifically, it focuses on the textile antenna system, which is that part of a wearable textile system that enables the transmission/reception of the RF-modulated data.

L. Vallozzi, P. Van Torre and H. Rogier are with the Information Technology Department (INTEC), Ghent University, St. Pietersnieuwstraat 41, 9000 Ghent, Belgium. E-mails: Luigi.Vallozzi@UGent.be Patrick.VanTorre@UGent.be Hendrik.Rogier@UGent.be

M. Moeneclaey is with the Department of Telecommunications and Information Processing (TELIN), Ghent University, St. Pietersnieuwstraat 41, 9000 Ghent, Belgium. E-mail: Marc.Moeneclaey@UGent.be

C. Hertleer is with the Department of Textiles, Ghent University, 9052 Zwijnaarde, Belgium. E-mail: Carla.Hertleer@UGent.be.

P. Van Torre and J. Verhaevert are with Hogeschool Gent, INWE department, Schoonmeersstraat 52, 9000 Ghent, Belgium
Wearable and textile antennas for body-centric communication have been the object of extensive research in the last years [3]-[6]. More recently, systems composed of several wearable or textile antennas have been investigated, proving the effectiveness of diversity techniques, both for on-body communication [7], [8] and for off-body communication [9], [10]. In this article, the specific case of communication between firefighter and base station is treated. In contrast to the existing literature, the proposed antenna system was designed in such a way in order to combat the following specific disturbances, that can make communication unreliable:

1) Firefighters often operate in indoor environments, characterized by rich multipath scattering of the signal, resulting in the received signal fading and consequent increase of the bit error rate;

2) The harsh environmental conditions in which firefighters often operate (i.e. high temperatures and/or high humidity) pose extra challenges in realizing the antennas with appropriate textile materials [4], [11], whose performance must not be affected by the severe environment;

3) The firefighter can optionally carry a metal oxygen bottle which may partially shield the antennas and restrict the suitable places where the antenna can be integrated into the jacket;

4) The close proximity of the human body to the antennas and the continuous deformation which they are subjected to, affect the designed radiation characteristics such as gain, resonance frequency and polarization, with a potential increase of the bit error rate.

The proposed wearable antenna system provides answers to the above needs by means of the following features:

1) To mitigate the severe fading of the indoor multipath environment, the antenna system uses pattern and polarization diversity, known to be effective diversity techniques [9], [12], [13]. This was realized by using two dual-polarized textile patch antennas [14], providing a total diversity order of $M=4$. Each antenna provides polarization diversity, while pattern diversity is added by placing one antenna at the front and the other at the rear of the body, thus creating two complementary hemispheres covering the complete $360^{\circ}$ azimuth range;

2) To prevent the antennas from being damaged by the extreme environmental conditions, the antenna substrate was realized in a protective, shock absorbing, fire retar- 
dant and water repellent foam, together with breathable conductive textile materials for patch and ground plane;

3) The two antennas were positioned in such a way in order to obtain minimum shielding or disturbance from the firefighter equipment, as it will be explained more in detail in the next sections;

4) Moreover, the antennas were placed at well-chosen locations in the jacket, where they are almost flat and not much subjected to large movements or bending due to the movements of the firefighter.

The performance and reliability of the realized system were tested during a measurement campaign, by means of a Signalion-HaLo 420 wireless testbed, which performs a real transmission of data allowing to determine the performance of the wireless link containing the textile antenna system.

The article is organized as follows: first, in Section II the textile antenna system is described, together with a complete description of the dual-polarized textile patch antenna, including the topology, design considerations and measured radiation characteristics such as gain patterns and polarization, in open space and on body. Section III-A describes the diversity settings and the combining techniques, while Section III-B describes the structure of the indoor environment. After that, in Sections III-C and III-D, the measurements conducted on the $2^{\text {nd }}$-order receiver polarization diversity and $4^{\text {th }}$-order receiver pattern-polarization diversity system are presented, together with a performance evaluation in terms of bit error rate and signal-to-noise ratio. Next, channel estimation and received signal correlation analysis are provided respectively in Sections III-E and III-F. After that, a more detailed analysis of bit error rate, including BERs after data detection and BER characteristics, is described in Sections III-G and III-H, respectively. Finally, in Section IV the conclusions are drawn.

\section{Textile Antenna System}

\section{A. Measurement Setup}

The measurement setup is composed of a transmitting vertically-polarized dipole antenna and the receiving wearable antenna system under test, which consists of two dualpolarized textile antennas, resulting in a total of four received diversity signals (two diversity signals per antenna). The proposed wearable antenna system is realized by integrating the two antennas into the front and back side of a firefighter jacket, worn by a test person, as shown in Fig. 1. The placement of the antennas inside the firefighter garment is shown in Fig. 2. All antennas are then connected to a Signalion-HaLo 420 measurement testbed, interfacing to Matlab by means of PCs. The measurement setup scheme is depicted in Fig. 3.

The transmitting unit of the testbed transmits bursts of QPSK data symbols modulating a carrier wave at $2.45 \mathrm{GHz}$. Note that in this measurement campaign we focus on QPSK modulation, in order to fix ideas. However, the measurement system allows the use of any modulation schemes, so the obtained results are easily generalized to other modulation schemes. A total of 244 bursts are transmitted, concatenating all measurement cases, as explained in Section III. Symbols are generated and modulated in Matlab and then upconverted

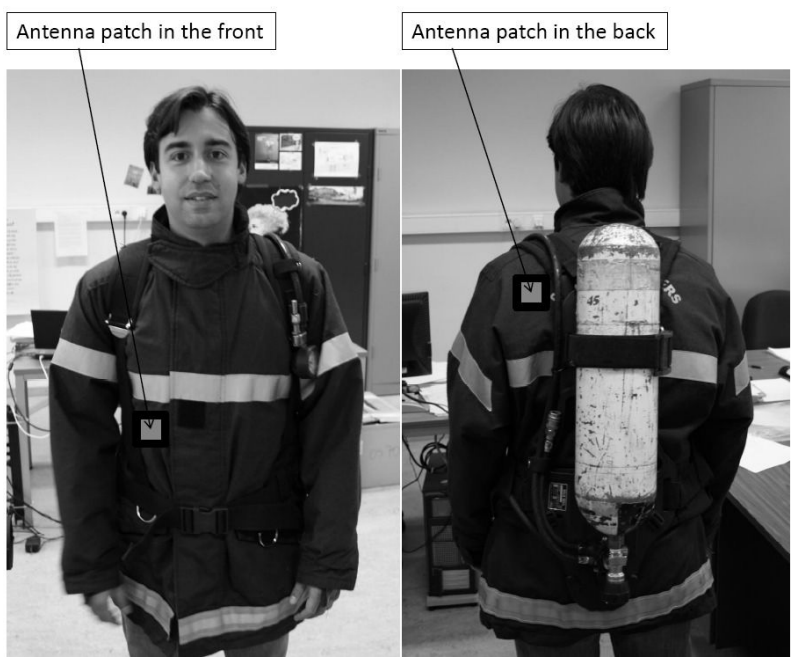

Fig. 1. Positions of front and rear antenna inside the jacket, covering two complementary azimuth hemispheres and ensuring minimal deformation of the antennas due to movement of the body.

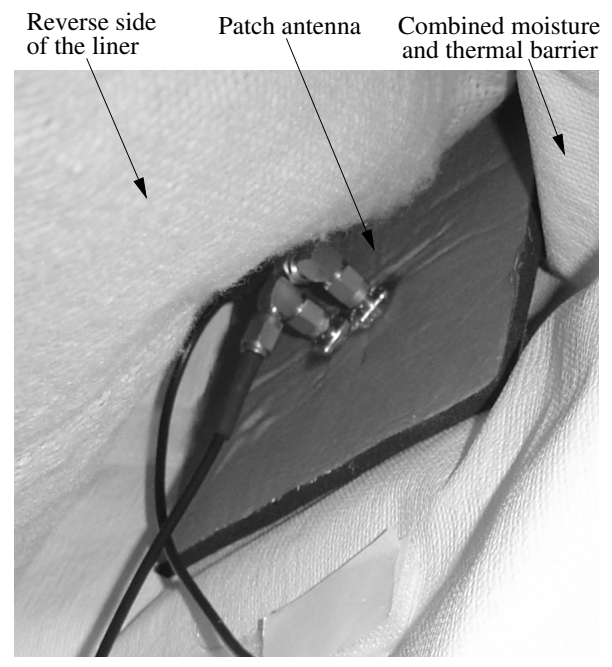

Fig. 2. Textile antenna inside the fireman's vest, behind the combined moisture and thermal barrier, protecting the antenna against harsh environmental conditions.

to RF by the testbed transmitter. The testbed receiver downconverts and samples the signals received by the textile antenna system. Demodulation, channel estimation, measurement and storage of the signal amplitude levels, diversity combining, detection and calculation of BER and SNR are performed in post processing on a PC by means of Matlab.

During the measurements, particular care was taken in placing the testbed at positions for which its effects on the propagation channel were reduced as much as possible.

\section{B. Structure and Radiation Characteristics of the Antennas}

The dual-polarized wearable antenna is a patch antenna completely made out of textile materials, suitable for integration into protective clothing such as firefighter suits. The substrate material is a protective, water-repellent, fire-retardant foam, commonly used in firefighter garments, whereas the ground plane and patch are made out of FlecTron ${ }^{\circledR}$ and 


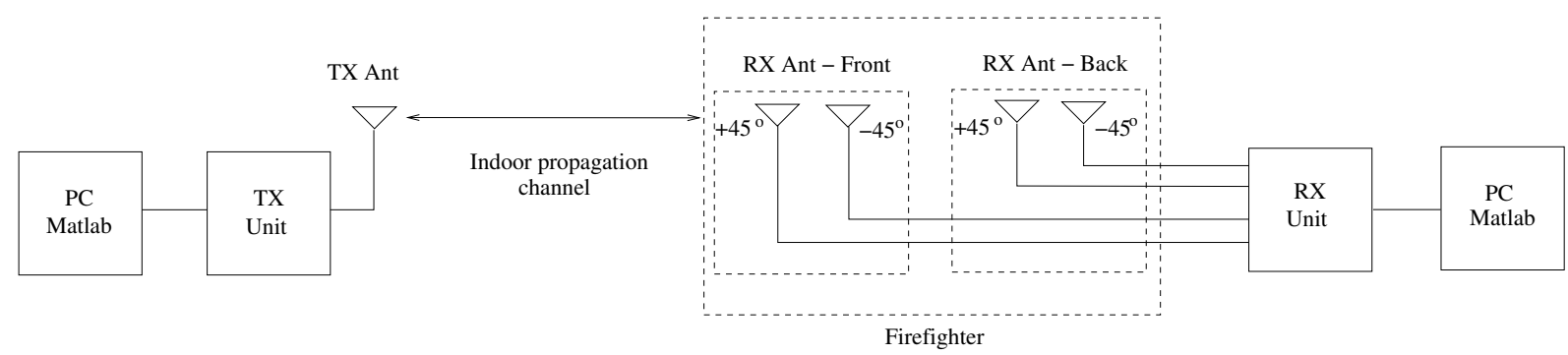

Fig. 3. Measurement setup scheme: communication link including transmitter, propagation channel and receiver implementing pattern-polarization diversity.

ShieldIt ${ }^{\circledR}$ respectively, two breathable and highly conductive textile materials.

The layout of the dual-polarized patch antenna consists of a rectangular patch with a slot. The antenna possesses two feed points, each one corresponding to an antenna terminal or port, located on the patch diagonals, symmetrically with respect to the Y-axis, as shown in the scheme in Fig. 4. The topology and feeding structure ensure the excitation of two signals with different polarizations, enabling the implementation of $2^{\text {nd }}$-order diversity in a compact single antenna system. The dimensions for this layout and the parameters for the used materials are listed in Fig. 4.
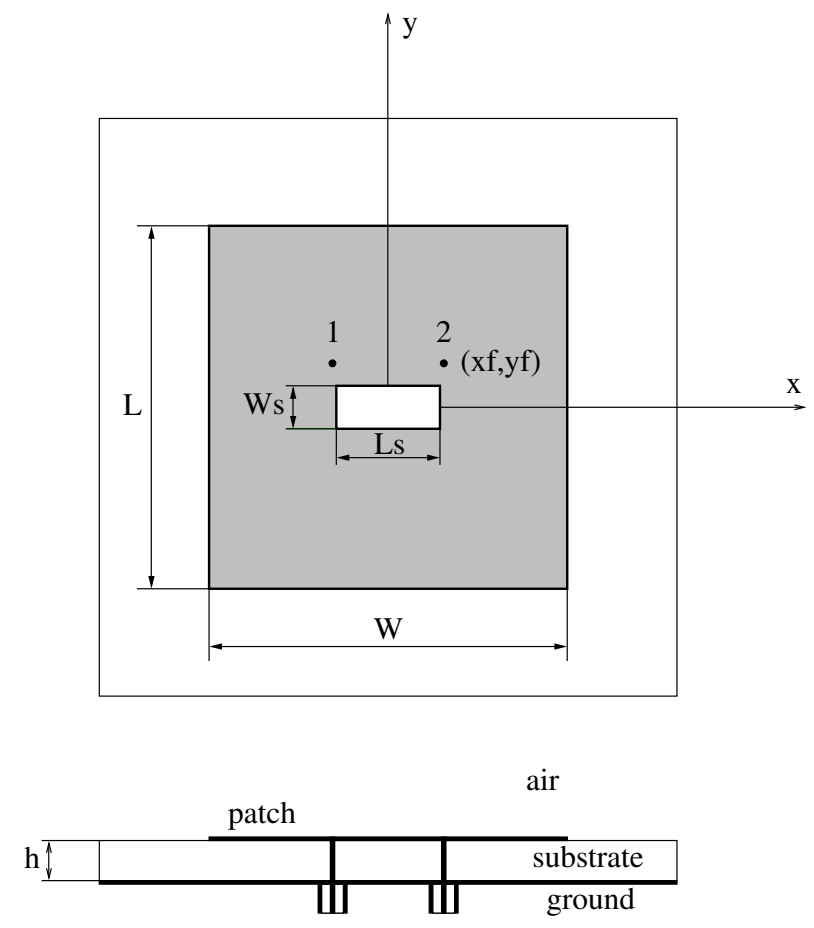

Fig. 4. Layout of the dual-polarized rectangular ring textile patch antenna. Feed points positioned symmetrically on the two diagonals, ensuring two orthogonal polarized waves. $L=49.59 \mathrm{~mm}, W=50.27 \mathrm{~mm}, L_{S}=14.34$ $\mathrm{mm}, W_{S}=5.94 \mathrm{~mm}$. Feed points positions: $\left( \pm x_{f}, \pm y_{f}\right)=( \pm 7.7 \mathrm{~mm}$, $\pm 6.08 \mathrm{~mm})$. Substrate parameters: $h=5.55 \mathrm{~mm}, \epsilon_{r}=1.12, \tan \delta=0.003$. The conductive materials are ShieldIt ${ }^{\circledR}$ (patch), FlecTron ${ }^{\circledR}$ (ground plane) and the substrate material is a protective closed-cell foam.

The measured radiation patterns of the antenna in open space, for both ports, are shown in Fig. 5 and 6. The radiation patterns were also measured while the antenna is embedded in the jacket, which is worn by a test person. Measurements

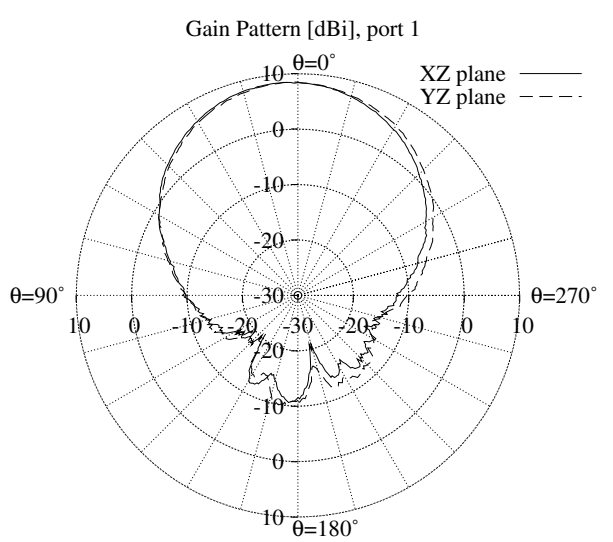

Fig. 5. Textile antenna in open space, measured radiation pattern

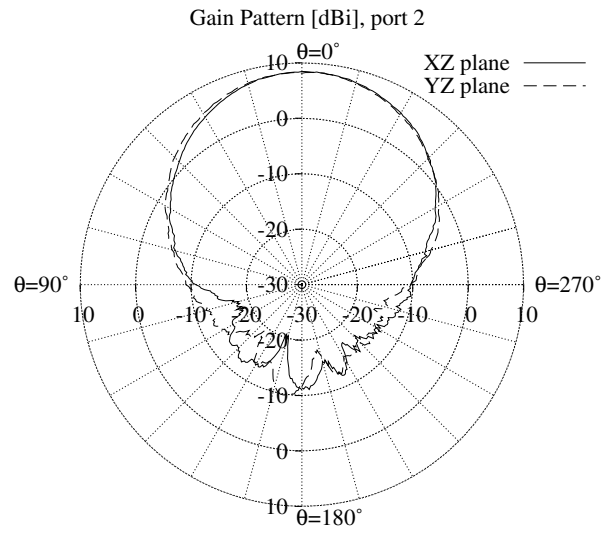

Fig. 6. Textile antenna in open space, measured radiation pattern

of the XZ-plane gain pattern with and without the oxygen cylinder carried on the back are compared in Figs. 7-10, for both ports of rear and front antenna, respectively. The oxygen bottle has a moderate influence on the gain patterns of rear and front antennas, deforming them and in some cases lowering the maximum gain. Very little power is radiated towards the body.

The antenna, in flat state, along broadside and at center frequency $f=2.45 \mathrm{GHz}$, was designed to transmit/receive two quasi-linearly polarized waves, which are almost orthogonal in space, with the two polarizations oriented at tilt angles of about $\alpha= \pm 45^{\circ}$. Each port signal radiates a field characterized by a radiation vector, expressed by the phasor 


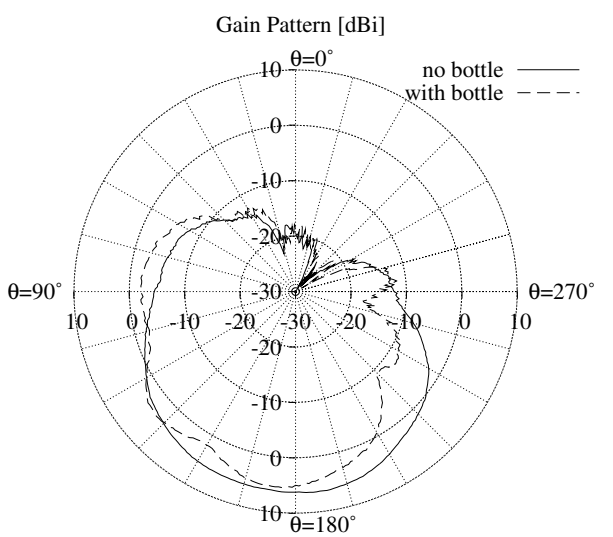

Fig. 7. Rear antenna on firefighter, port 1, measured XZ-plane gain pattern. The presence of the metal oxygen bottle, carried by the firefighter, produces deformation of the gain pattern and lowering of the maximum gain.

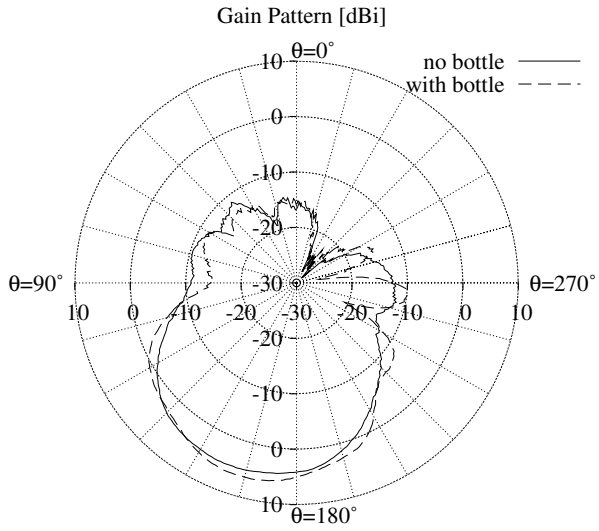

Fig. 8. Rear antenna on firefighter, port 2, measured XZ-plane gain pattern. The presence of the metal oxygen bottle, carried by the firefighter, produces deformation of the gain pattern.

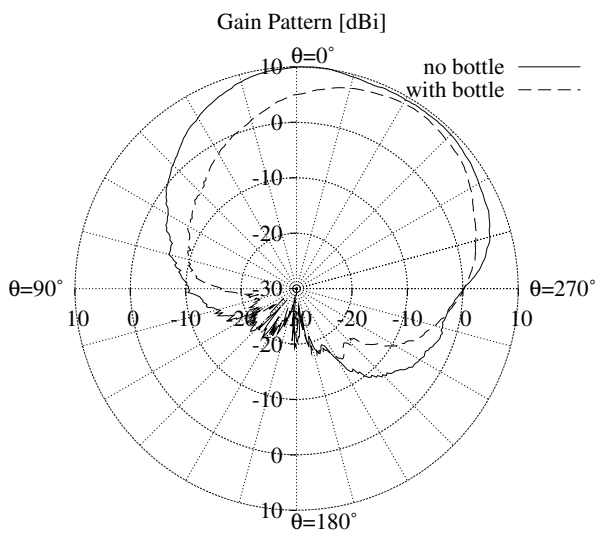

Fig. 9. Front antenna on firefighter, port 1, measured XZ-plane gain pattern. The presence of the metal oxygen bottle, carried by the firefighter, produces deformation of the gain pattern and lowering of the maximum gain.

vector $\mathbf{F}=A\left(\hat{u}_{x}-j \tau \hat{u}_{y}\right)$. This vector rotates in time, when the signal is propagating, tracing a polarization ellipse, which completely describes the polarization of the signal on the XYplane, which is characterized by the following polarization parameters:

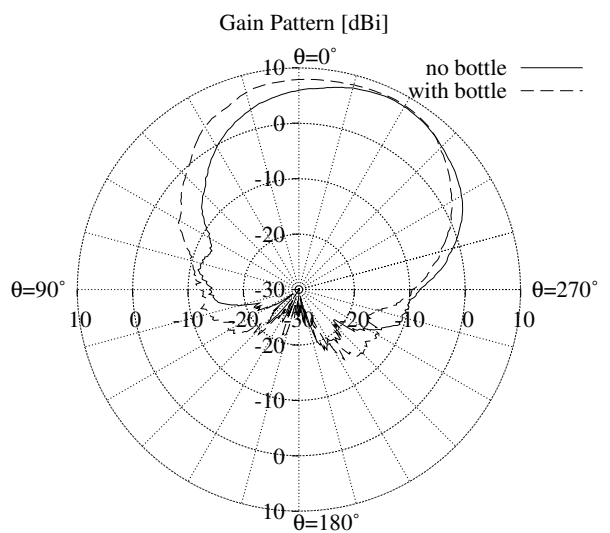

Fig. 10. Front antenna on firefighter, port 2, measured XZ-plane gain pattern. The presence of the metal oxygen bottle, carried by the firefighter, produces deformation of the gain pattern.

- Tilt angle $\alpha$, that is the angle between the $\mathrm{X}$-axis of the antenna (as shown in Fig. 4) and the major axis of the polarization ellipse;

- Eccentricity $\tau$, that is the ratio between the minor and the major axis of the polarization ellipse;

- Axial ratio for linear polarization (which provides the same information as $\tau$ ), defined as $A R_{L P}=20$. $\log _{10}[(1+|\tau|) /(1-|\tau|)]$.

The polarization parameters for both port signals were measured in open space and with the antenna worn by the test person at the front and back, with and without the oxygen cylinder present. Measurements of the tilt angle, eccentricity and axial ratio for linear polarization, for the broadside direction, are listed in Tables I - III. For each listed case, a coefficient describing the orthogonality of the polarization ellipses of the two ports, being $Q_{12}$, is also given. $Q_{12}$ is defined by:

$$
\begin{aligned}
Q_{12} & \triangleq \frac{\left|\mathbf{F}_{1} \cdot \mathbf{F}_{2}^{*}\right|^{2}}{\left|\mathbf{F}_{1}\right|^{2}\left|\mathbf{F}_{2}\right|^{2}}= \\
& =\frac{\left(\tau_{1}+\tau_{2}\right)^{2}}{\left(1+\tau_{1}^{2}\right)\left(1+\tau_{2}^{2}\right)}+\cos ^{2}\left(\alpha_{1}-\alpha_{2}\right) \frac{\left(1-\tau_{1}^{2}\right)\left(1-\tau_{2}^{2}\right)}{\left(1+\tau_{1}^{2}\right)\left(1+\tau_{2}^{2}\right)}
\end{aligned}
$$

with $0 \leq Q_{12} \leq 1$. On the one hand, if $Q_{12}=0$ the two polarization ellipses are orthogonal, thus independent. On the other hand, if $Q_{12}=1$ the two ellipses are coincident. For a dual-polarized antenna the desired value is $Q_{12}=0$, in order to have minimal correlation between the two port signals.

\begin{tabular}{r|r|r|r} 
Port & $\alpha$ & $\tau$ & $A R_{L P}$ \\
\hline 1 & $45.56^{\circ}$ & -0.02 & 0.41 \\
2 & $130.73^{\circ}$ & 0.01 & 0.19 \\
\multicolumn{3}{|c}{$Q_{12}=0.007$}
\end{tabular}

TABLE I

ORThogonality of the Antenna Ports In Open SPaCe

From the measurements, we conclude that:

- Compared to the open-space case, when the antenna is worn inside the jacket on a human body (with and without bottle), the polarization departs from being linear 


\begin{tabular}{r|r|r|r||r|r|r} 
& \multicolumn{3}{|c||}{ Without $\mathrm{O}_{2}$} & \multicolumn{3}{c}{ With $\mathrm{O}_{2}$} \\
\hline Port & $\alpha$ & $\tau$ & $A R_{L P}$ & $\alpha$ & $\tau$ & $A R_{L P}$ \\
\hline 1 & $64.42^{\circ}$ & -0.24 & 4.16 & $66.94^{\circ}$ & -0.60 & 11.94 \\
2 & $69.03^{\circ}$ & 0.74 & 16.39 & $32.10^{\circ}$ & 0.68 & 14.39 \\
& \multicolumn{3}{|c|}{$Q_{12}=0.41$} & \multicolumn{3}{c}{$Q_{12}=0.12$}
\end{tabular}

TABLE II

Orthogonality of the Antenna Ports of the Front Antenna on THE BODY

\begin{tabular}{r|r|r|r||r|r|r} 
& \multicolumn{3}{|c||}{ Without $\mathrm{O}_{2}$} & \multicolumn{3}{c}{ With $\mathrm{O}_{2}$} \\
\hline Port & $\alpha$ & $\tau$ & $A R_{L P}$ & $\alpha$ & $\tau$ & $A R_{L P}$ \\
\hline 1 & $11.37^{\circ}$ & -0.65 & 13.50 & $147.63^{\circ}$ & -0.53 & 10.18 \\
2 & $108.14^{\circ}$ & 0.11 & 1.91 & $61.62^{\circ}$ & 0.22 & 3.86 \\
& \multicolumn{3}{|c||}{$Q_{12}=0.21$} & \multicolumn{3}{c}{$Q_{12}=0.07$}
\end{tabular}

TABLE III

ORthogonality of the Antenna Ports of the BACK ANTENNA ON THE BODY

to become elliptical. This is seen from the values for $\tau$ or $A R_{L P}$;

- In some cases the presence of the oxygen bottle also produces a slight rotation of the polarization ellipses and changes the axial ratios, having an influence on the signal correlation;

- When the antenna is worn, for both front and back antenna and with and without oxygen bottle, the orthogonality coefficient $Q_{12}$ increases with respect to the openspace situation, ranging from a minimum of 0.07 to a maximum of 0.41 . This clearly indicates that the integration of the antennas into the jacket and the presence of the bottle has a negative effect on the orthogonality of the two ports signals, increasing their correlation. This effect is further studied in Section III.

Additional effects of bending and deformation of the textile antennas are not discussed in this article, as these were already investigated in previous publications. In [14] is shown that for a dual-polarized textile patch antenna, subjected to bending, the return loss remains acceptable over the frequency band of interest. Moreover, in [4] is proved that, for a single circularly polarized antenna, the return loss, gain and axial ratio are still satisfactory under bending.

\section{Receiver Diversity Using Dual Polarized TEXTILE ANTENNAS}

\section{A. Diversity Schemes and Combining Techniques}

In this paper, we focus on receiver diversity employed by the off-body antenna system. We do not consider diversity at the base station/access point side, as this is easier to implement. We considered two different receiver-diversity schemes:

- $2^{\text {nd }}$-order receiver polarization diversity, i.e. with one transmitting dipole on the test bed and one receiving dualpolarized antenna (front or back) on the firefighter.

- $4^{\text {th }}$-order receiver combined pattern-polarization diversity, i.e. with one transmitting dipole on the testbed and two receiving dual-polarized antennas (front and back) on the firefighter.
Moreover, for each diversity scheme, two different combining schemes were employed: Maximal ratio combining (MRC) and Selection combining (SC). In all considered cases, performance analysis demonstrated the advantage of using diversity for the wearable antenna system.

\section{B. The Indoor Environment}

A floor plan of the indoor environment where the measurements were performed is displayed in Fig. 11. The path followed by the test person during the measurements is marked, as well as the position of the transmitter. The considered cases are listed here as a function of the labels shown in Fig. 12, 13 and 14, in Sections III-C and III-D.

1) Path $A_{1} \rightarrow B_{1}$ : the test person walking towards the transmitter from a distance of $15 \mathrm{~m}$ and ending at $3 \mathrm{~m}$ from the transmitter;

2) Path $B_{1} \rightarrow A_{2}$ : walking away from the transmitter, in the opposite direction of the first path;

3) Path $A_{2} \rightarrow B_{2} \rightarrow A_{3}$ : walking towards the transmitter and returning, i.e. the two previous paths combined in one measurement series;

4) Path $A_{3} \rightarrow C_{1} \rightarrow A_{4}$ : walking sideways, along a path perpendicular to the transmitter, at up to $18 \mathrm{~m}$ of distance. This is the hardest receiving path, since the distance is large and there are many obstacles in the signal path.

The firefighter can optionally carry a metal oxygen bottle on the back. Measurements presented in Section II-B suggest a minor performance degradation due to the presence of the bottle. For that reason, to test the worst-case scenario, the following measurements were performed with the oxygen bottle present.

\section{C. $2^{\text {nd }}$-Order Receiver Diversity Using One Dual-Polarized Textile Antenna}

The indoor environment where the measurements were performed is characterized by a high amount of multipath propagation, resulting in small-scale fading. For the walking firefighter the signal levels are also influenced by shadowing and changing path loss.

In order to investigate the use of polarization diversity to mitigate fading effects, $2^{\text {nd }}$-order receiver diversity based on a single dual-polarized antenna was first implemented. At the transmitting unit a transmit power of $-10 \mathrm{dBm}$ was applied. Symbols were transmitted using Quadrature Phase Shift Keyed (QPSK) modulation and organized in sequential bursts. The symbol rate was 1 MSymbol per second, corresponding to a bit rate of $2 \mathrm{MBit} / \mathrm{s}$. Each burst is composed of 300 pilot symbols for channel estimation, followed by 396 data symbols and it has a total duration of $696 \mu$ s, which is short enough to consider the channel invariant during one burst. At the receiver side, MRC and SC were applied to the two output signals coming from a single patch antenna.

In Fig. 12 and 13 the signal-to-noise ratios corresponding to each of the two polarizations are displayed, together with the bit error rates obtained using MRC and SC. The labels on 


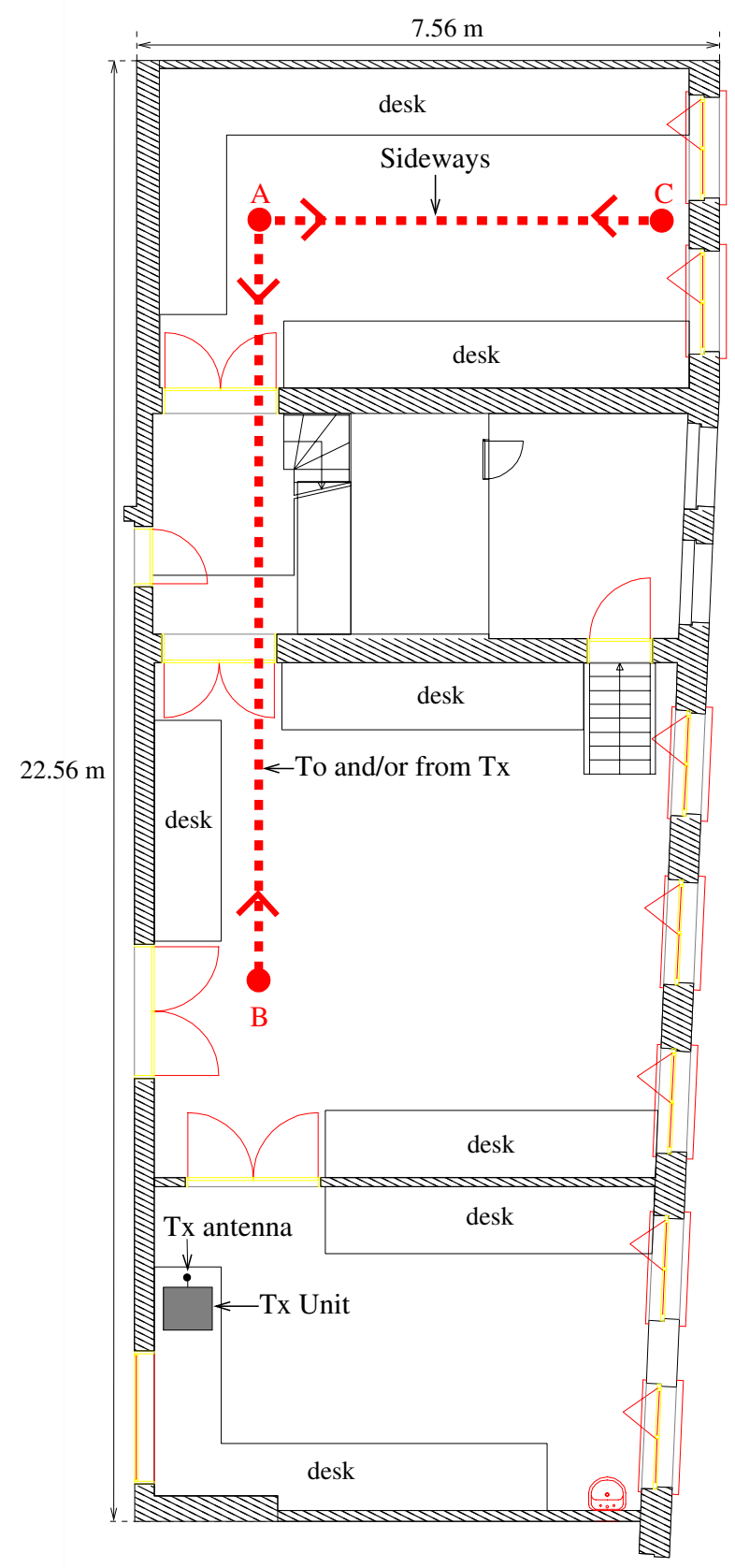

Fig. 11. Floor plan of the indoor environment. The position of the fixed TX unit is shown together with the paths walked by the test person: walking to and/or from TX (A-B) and walking sideways (A-C).

top of the graphs correspond to the paths walked, as described for the floor plan.

One notices that, for both front and rear antennas, the signal-to-noise ratios increase as the test person is approaching the transmitter and decrease when walking away from the transmitter (cases 1,2,3). In the sidewalk path (case 4), when there is no line of sight, the SNR has a constant mean value, since the scattered rays are dominant compared to the direct ray.

As far as the bit errors are concerned, by using MRC, the BER decreases significantly compared to the BER for each of the original signals, the average BER being reduced from an order of about $10^{-2}$ with a single polarization, to about $10^{-3}$ with dual polarization, using SC or MRC. Thus, application of polarization diversity with a dual polarized antenna clearly results in improvement of the communication quality. A more detailed analysis of the BER, together with the achieved diversity gain, is provided in Section III-H.

Moreover, the measurements using polarization diversity indicate that both antenna signals fade independently as bit errors often occur at different times for each signal. This reveals low correlation between the two diversity signals received by the antenna, as confirmed by the correlation analysis, presented in Section III-F.

\section{D. $4^{\text {th }}$-Order Receiver Diversity Using Two Dual-Polarized Textile Antennas}

Compared to the $2^{\text {nd }}$-order polarization diversity with only one receiving antenna (front or back), additional improvement is expected by exploiting $4^{\text {th }}$-order pattern-polarization diversity using two dual-polarized antennas, i.e. using the four signals coming from both the front and rear antennas.

Also exploiting pattern diversity is advantageous for several reasons. First, because the firefighter can block the path between the transmitter and receiver antenna, i.e. his body shields the antenna that is oriented away from the transmitter. Moreover, the directional radiation pattern of the patch antennas increases the influence of the orientation. Because of these effects, the signal is stronger for the front antenna (compared to the rear antenna) when approaching the transmitter and vice versa when walking away from the transmitter. However the antenna oriented away from the transmitter still receives an acceptable signal caused by the multipath propagation of the signals. The hardest path is the sideways track, at a large distance from the transmitter (for the $0.1 \mathrm{~mW}$ transmitted power) and with many obstacles in between.

In order to have significant advantage from the $4^{\text {th }}$-order diversity, sufficiently low correlations are necessary between the four signals. In Fig. 14 the different SNR curves show that the fading patterns are different for the four signals, suggesting low correlations. Especially the front and rear signals fade independently over a large period of time.

The performance investigation was repeated for the $4^{\text {th }}$-order diversity case in the same way as for the $2^{\text {nd }}$-order diversity case. The resulting measured SNR and computed BER, using MRC and SC, are shown in Fig. 14. Combining the four signals with SC or MRC shows a significant improvement in performance, here pattern and polarization diversity are combined. In our case no errors are left after applying MRC to the signals and detecting the data.

\section{E. Channel Estimations}

The average channel estimations for both antennas and for the combined signals also allow to evaluate the effectiveness of receiver diversity. To estimate the gain obtained by using polarization diversity, MRC is considered for the two signals received by the same patch antenna. The gain obtained by combining 4 signals (pattern and polarization diversity together) is also listed, for MRC and SC. 


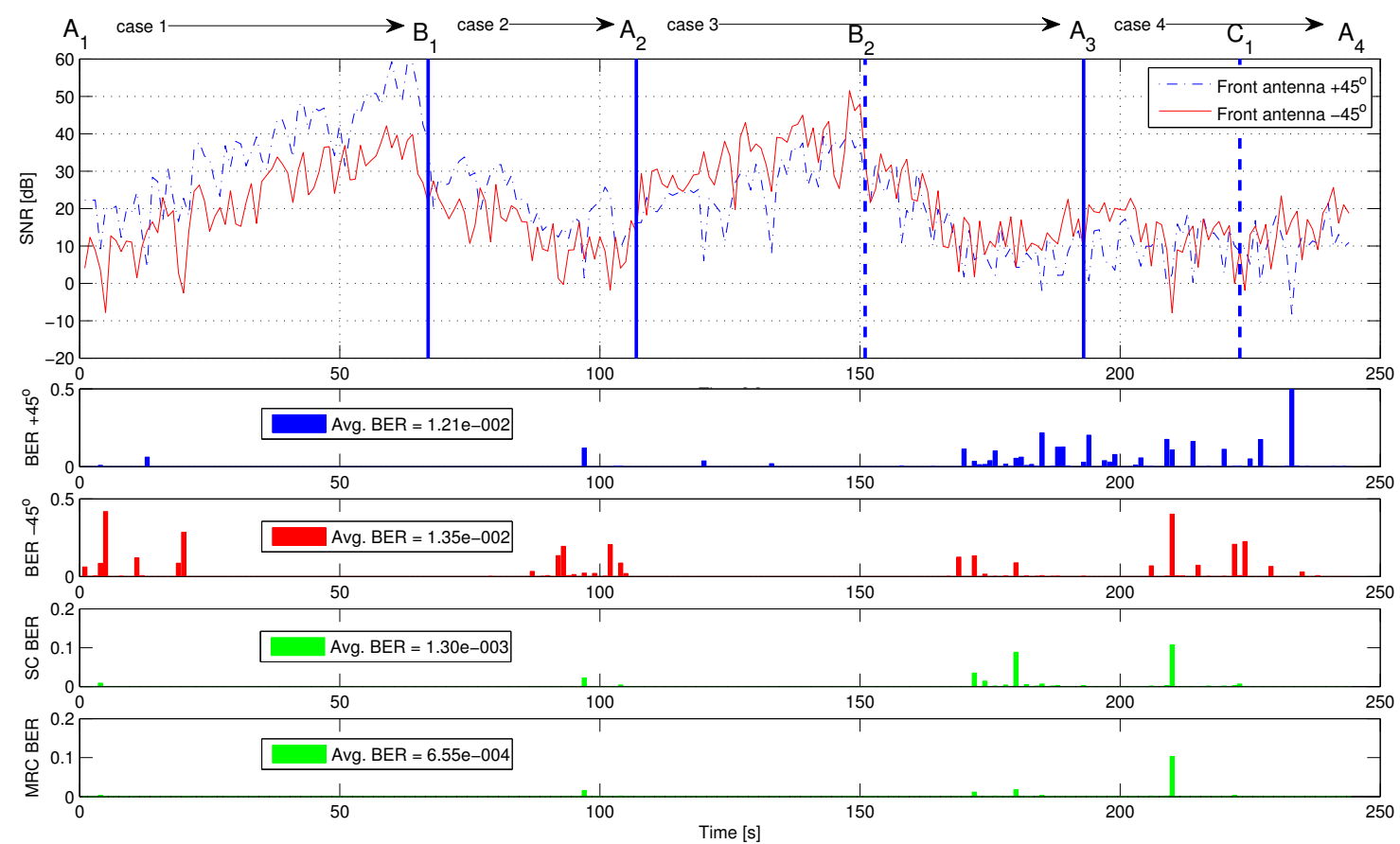

Fig. 12. Received SNR, BER for each polarization, as well as BER after SC and MRC, for the front antenna on the walking firefighter equipped with a metal oxygen bottle strapped on the back. Application of polarization diversity with a single dual-polarized textile antenna, with SC or MRC, results in a drastic reduction of BER.

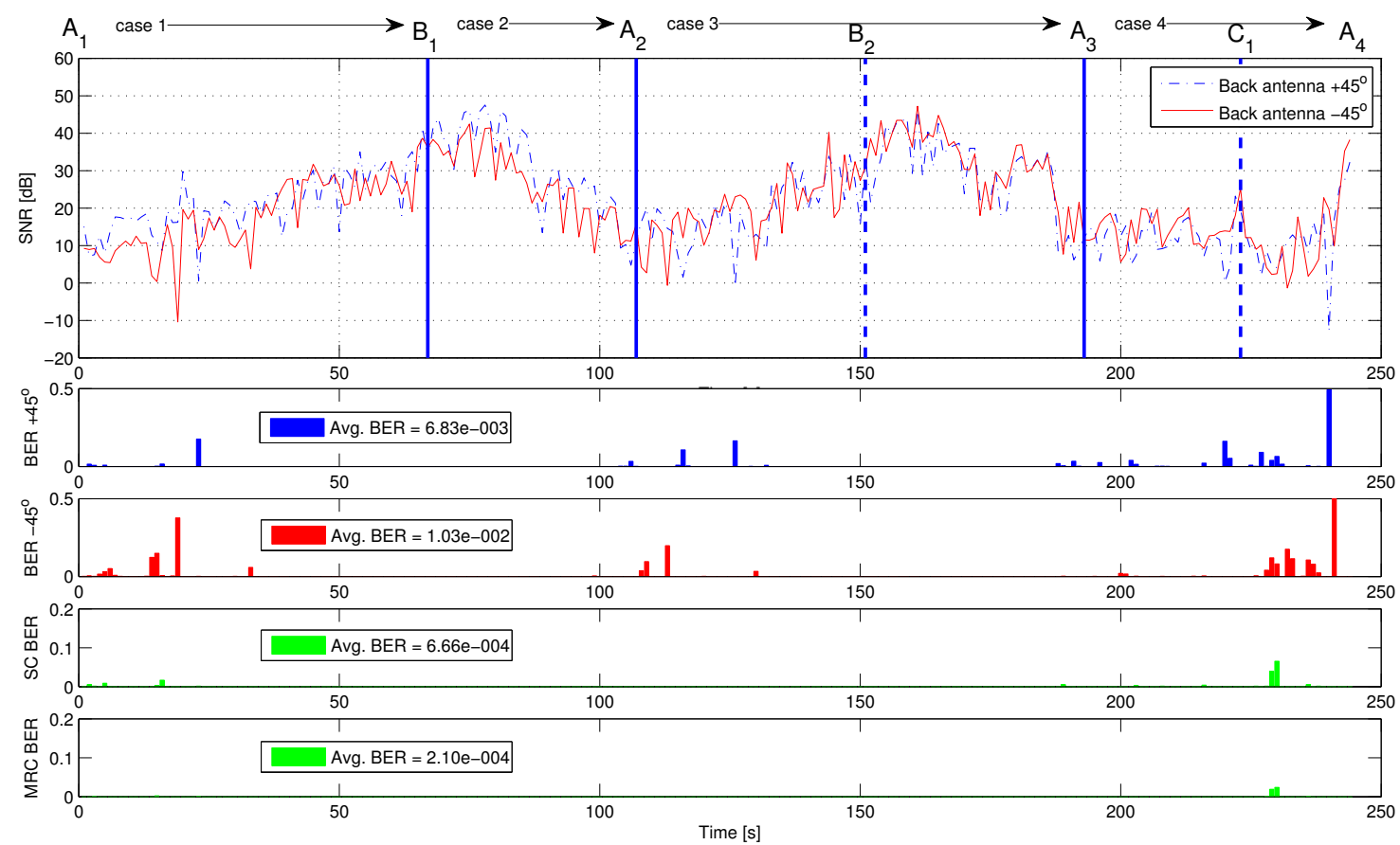

Fig. 13. Received SNR, BER for each polarization, as well as BER after SC and MRC, for the back antenna on the walking firefighter equipped with a metal oxygen bottle strapped on the back. Application of polarization diversity with a single dual-polarized textile antenna, with SC or MRC, results in a drastic reduction of BER.

Given the $h_{1}$ to $h_{4}$ being the random variables representing the channel estimates for each complete set of measurements corresponding to the different cases, the average channel estimations were calculated as following.

- $10 \cdot \log \left[E\left(\left|h_{1}\right|^{2}\right)\right]$ Back antenna, channel 1; 


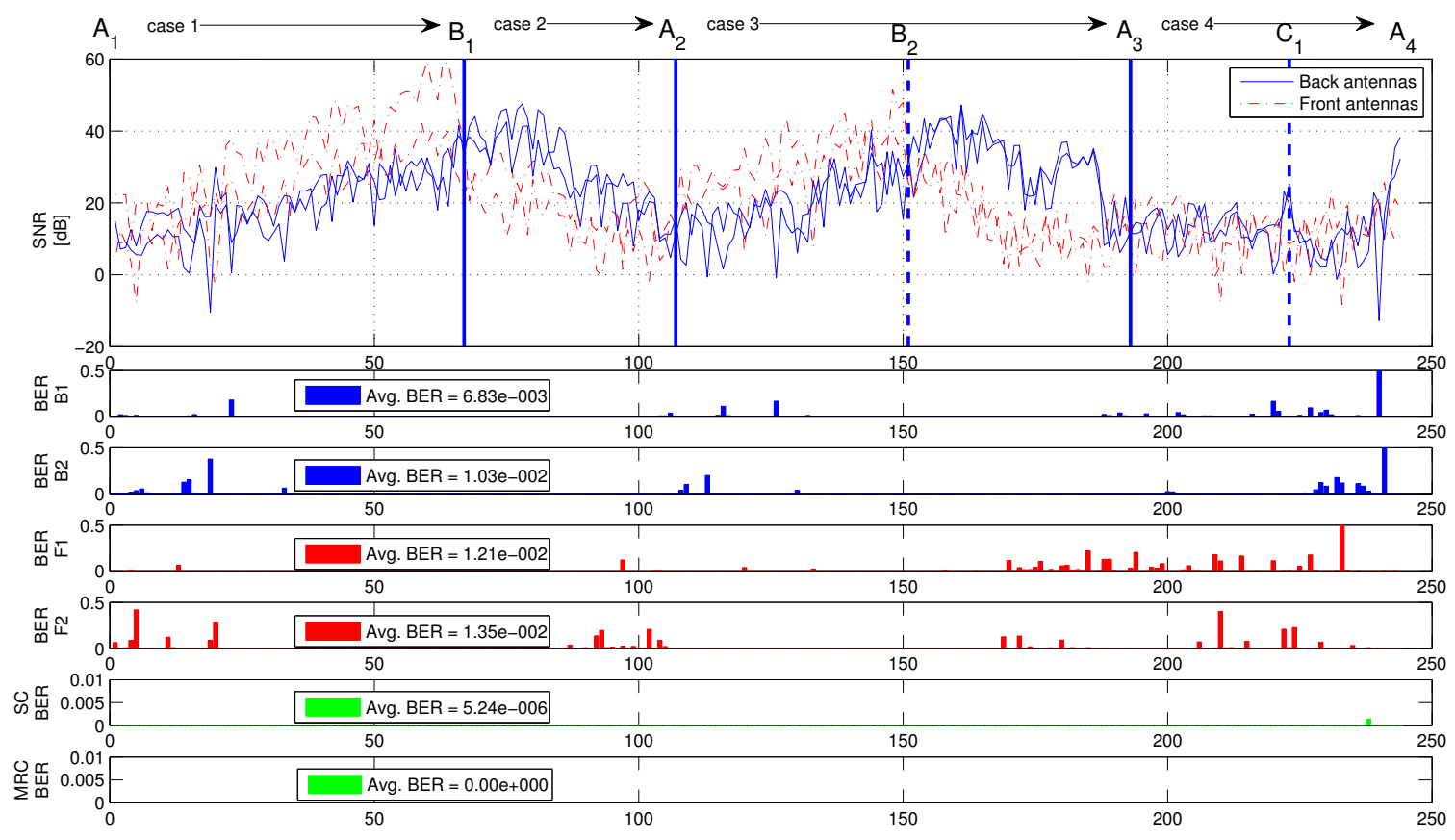

Fig. 14. Received SNR, BER for each antenna, as well as BER after SC and MRC, for a walking firefighter walking with a metal oxygen bottle strapped on the back. Application of pattern-polarization diversity with two dual-polarized textile antennas results in a large improvement of the quality. No errors are left after detection, with both SC or MRC.

- $10 \cdot \log \left[E\left(\left|h_{2}\right|^{2}\right)\right]$ Back antenna, channel 2;

- $10 \cdot \log \left[E\left(\left|h_{1}\right|^{2}+\left|h_{2}\right|^{2}\right)\right] \operatorname{MRC} 1,2$;

- $10 \cdot \log \left[E\left(\left|h_{3}\right|^{2}\right)\right]$ Front antenna, channel 3;

- $10 \cdot \log \left[E\left(\left|h_{4}\right|^{2}\right)\right]$ Front antenna, channel 4;

- $10 \cdot \log \left[E\left(\left|h_{3}\right|^{2}+\left|h_{4}\right|^{2}\right)\right] \operatorname{MRC} 3,4$;

- $10 \cdot \log \left[E\left(\left|h_{1}\right|^{2}+\left|h_{2}\right|^{2}+\left|h_{3}\right|^{2}+\left|h_{4}\right|^{2}\right)\right]$ MRC 1-4;

- $10 \cdot \log \left[E\left(\max \left(\left|h_{1}\right|^{2},\left|h_{2}\right|^{2},\left|h_{3}\right|^{2},\left|h_{4}\right|^{2}\right)\right)\right]$ SC 1-4.

In the above expressions $E(\cdot)$ indicates the statistical expectation, calculated as an arithmetical average over the total available $N$ samples, representing $N$ realizations of the corresponding random variable.

The average is calculated for each scenario (cases 1-4, as defined in Section III-B), together with the average signal levels resulting from different combining schemes. The results for the different measurement paths, converted to $\mathrm{dB}_{\mu \mathrm{v}}$ on a $50 \Omega \mathrm{load}$, are listed in Table IV. For the interpretation of the values in this table, the combined signals should be compared to all corresponding input signals, not only to the best input signal involved in the calculation. When using only one antenna, in a real-life situation, the received signal will sometimes correspond to the worst input signal.

From this table it is clear that polarization diversity by itself provides a significant gain in average signal level. By MRC combining 4 signals, adding pattern diversity, the gain is always significantly larger.

Comparing the difference in average signal levels for the

\begin{tabular}{c||c|c|c|c|c}
\multicolumn{1}{c||}{ Channel } & \multicolumn{5}{c}{ Case nr. } \\
\cline { 5 - 7 } & 1 & 2 & 3 & 4 & all $(1-4)$ \\
\hline$h_{1}$ & 18.15 & 32.39 & 26.25 & 7.21 & 26.55 \\
$h_{2}$ & 16.55 & 25.08 & 27.00 & 14.10 & 23.93 \\
MRC $h_{1}, h_{2}$ & 20.44 & 33.13 & 29.65 & 14.91 & 28.45 \\
\hline$h_{3}$ & 42.97 & 14.75 & 20.31 & -1.67 & 37.39 \\
$h_{4}$ & 22.62 & 6.06 & 30.41 & 4.06 & 26.42 \\
MRC $h_{3}, h_{4}$ & 43.01 & 15.30 & 30.82 & 5.09 & 37.72 \\
\hline MRC $h_{1}-h_{4}$ & 43.03 & 33.20 & 33.28 & 15.34 & 38.21 \\
SC $h_{1}-h_{4}$ & 42.97 & 32.40 & 32.19 & 14.47 & 38.00 \\
\multicolumn{5}{c}{ TABLE IV }
\end{tabular}

Average Channel Estimates (Signal LeVel in DB $\mu V$ ON A $50 \Omega$ LOAD) FOR DIFFERENT DIVERSITY SCENARIOS

four inputs reveals that the difference between front and back signals can be much larger than the difference between signals received on the same patch antenna, differing only in polarization. This behavior is expected, taking into account the shadowing by the body and the directional radiation pattern of the antennas. The use of pattern diversity combined with polarization diversity provides the largest gain when a direct signal path is present, in that case the antenna oriented to the transmitter will consistently provide stronger signals. However, also in the absence of a direct path the use of all four signals provides a significant performance gain. Bit-error-rate graphs presented in Section III-H will confirm that an effective fourth order diversity is obtained.

From a hardware point of view, SC of the signals is a simpler way to obtain diversity but performs up to $2 \mathrm{~dB}$ worse than MRC in our measurements. 
The average values only partially display the potential performance gain resulting from diversity. Because the variance for the combined signals will be smaller than for the individual signals, a lower bit error rate will be obtained for the same average signal level. The bit-error-rate characteristics in Sections III-G and III-H further demonstrate this additional performance improvement.

\section{F. Correlation Between the Signals}

The correlation coefficient matrices were calculated based on the channel estimations corresponding to each signal. The correlation coefficients are determined for a sliding window of 10 measurements at a time. The reason why this method was adopted, is to account for the fading only and not for shadowing or path loss, as the latter two parameters are assumed to be constant within the used window of 10 channel estimates.

Given $X=\left|h_{1}\right|^{2}$ and $Y=\left|h_{2}\right|^{2}$, scalar random variables representing the squared channel gains, and $E(\cdot)$ indicating the statistical expectation, the correlation coefficient is given by

$$
\rho_{X, Y}=\frac{E(X Y)-E(X) E(Y)}{\sqrt{\left[E\left(X^{2}\right)-(E(X))^{2}\right]\left[E\left(Y^{2}\right)-(E(Y))^{2}\right]}}
$$

In practice, the statistical expectations in the right hand side of (1) were calculated as arithmetical averages over $K=10$ samples contained in a window, representing 10 realizations of the random variables.

The correlation coefficients obtained in this way are then averaged to provide the final correlation value. A direct calculation of the correlation coefficient based on the complete measurement series often results in low correlation values that are not necessarily related to the performance of the communication system. Especially the shadowing of the body, combined with changes in orientation of the firefighter, can result in altering statistics for both antennas during the same measurement series. Within the window of 10 measurements used, the statistics of the signals do not vary significantly.

The results are displayed in Tables V to IX. Antennas F1 and $\mathrm{F} 2$ correspond to the two polarizations for the front antenna, likewise B1 and B2 are the signals for the back antenna. For front and back signals the correlation is very low, hence using two antennas improves the performance of the system a lot. The gain obtained by using front and back antennas is not only related to fading, as the radiation pattern of the antennas is directional and the front and back antennas each cover approximately a complementary hemisphere around the body.

The correlation is higher for signals differing only in polarization compared to the values for front and back antennas. However polarization diversity is still present to a varying degree depending on the situation. The bit-error-rate measurements in the next section will confirm that a significant performance gain can be obtained by adding polarization diversity.

\begin{tabular}{r|rrrr} 
Antenna & \multicolumn{1}{|c}{ F1 } & \multicolumn{1}{c}{ F2 } & \multicolumn{1}{c}{ B1 } & \multicolumn{1}{c}{ B2 } \\
\hline F1 & 1.0000 & 0.2626 & -0.0189 & 0.0768 \\
F2 & 0.2626 & 1.0000 & 0.1476 & -0.1771 \\
B1 & -0.0189 & 0.1476 & 1.0000 & 0.2657 \\
B2 & 0.0768 & -0.1771 & 0.2657 & 1.0000 \\
& \multicolumn{4}{|}{ TABLE V }
\end{tabular}

Correlation of the Received Signal Power Between the ANTENNAS FOR THE WALK TO THE TRANSMITTER.

\begin{tabular}{r|rrrr} 
Antenna & \multicolumn{1}{|c}{ F1 } & \multicolumn{1}{c}{ F2 } & \multicolumn{1}{c}{ B1 } & \multicolumn{1}{c}{ B2 } \\
\hline F1 & 1.0000 & 0.5473 & -0.0612 & 0.0200 \\
F2 & 0.5473 & 1.0000 & 0.1229 & -0.0664 \\
B1 & -0.0612 & 0.1229 & 1.0000 & 0.1808 \\
B2 & 0.0200 & -0.0664 & 0.1808 & 1.0000 \\
\multicolumn{5}{c}{ TABLE VI }
\end{tabular}

Correlation of the Received Signal Power Between the AntenNas For the Walk AWAy From the TransmitTer.

\begin{tabular}{r|cccc} 
Antenna & F1 & F2 & B1 & B2 \\
\hline F1 & 1.0000 & 0.5352 & 0.1046 & 0.1172 \\
F2 & 0.5352 & 1.0000 & 0.0849 & 0.0406 \\
B1 & 0.1046 & 0.0849 & 1.0000 & 0.2450 \\
B2 & 0.1172 & 0.0406 & 0.2450 & 1.0000 \\
\multicolumn{5}{c}{ TABLE VII }
\end{tabular}

Correlation of the Received Signal Power Between the ANTENNAS FOR THE WALK TO AND RETURN FROM THE TRANSMITTER Without OXYGEN BOTTLE.

\begin{tabular}{r|rrrr} 
Antenna & \multicolumn{1}{|c}{ F1 } & \multicolumn{1}{c}{ F2 } & \multicolumn{1}{c}{ B1 } & \multicolumn{1}{c}{ B2 } \\
\hline F1 & 1.0000 & 0.3638 & -0.0972 & -0.2886 \\
F2 & 0.3638 & 1.0000 & -0.0446 & -0.1223 \\
B1 & -0.0972 & -0.0446 & 1.0000 & 0.1797 \\
B2 & -0.2886 & -0.1223 & 0.1797 & 1.0000
\end{tabular}

TABLE VIII

Correlation of the Received Signal Power Between the ANTENNAS FOR THE WALK SIDEWAYS TO THE TRANSMITTER.

\begin{tabular}{r|rrrr} 
Antenna & \multicolumn{1}{|c}{ F1 } & \multicolumn{1}{c}{ F2 } & \multicolumn{1}{c}{ B1 } & \multicolumn{1}{c}{ B2 } \\
\hline F1 & 1.0000 & 0.4227 & -0.0027 & 0.0013 \\
F2 & 0.4227 & 1.0000 & 0.0637 & -0.0783 \\
B1 & -0.0027 & 0.0637 & 1.0000 & 0.2225 \\
B2 & 0.0013 & -0.0783 & 0.2225 & 1.0000
\end{tabular}

TABLE IX

Correlation of the Received Signal Power Between the ANTENNAS FOR ALL THE PREVIOUS SCENARIOS TOGETHER.

\section{G. Bit Error Rates After Data Detection}

The bit error rates for all measurements combined are shown in table X. These values have been determined by demodulating the received signal, detecting the data and counting the number of errors.

The values clearly illustrate the performance gain obtained by using maximal ratio combining of the signals. The values obtained by combining the two orthogonally polarized signals from the same antenna already show a significant diversity gain.

In this experiment and for the limited number of symbols transmitted, using maximal ratio combining with all four sig- 


\begin{tabular}{l|c}
\multicolumn{1}{c|}{ Signal } & BER \\
\hline Back antenna signal 1 & $6.8 \cdot 10^{-3}$ \\
Back antenna signal 2 & $1.0 \cdot 10^{-2}$ \\
MRC of both back signals & $2.1 \cdot 10^{-4}$ \\
Front antenna signal 1 & $1.2 \cdot 10^{-2}$ \\
Front antenna signal 2 & $1.4 \cdot 10^{-2}$ \\
MRC of both front signals & $6.6 \cdot 10^{-4}$ \\
Maximal Ratio Combining 4 signals & 0 \\
Selection Combining 4 signals & $5.2 \cdot 10^{-6}$
\end{tabular}

TABLE X

Bit ERROR RATES FOR DiFFERENT RECEIVED AND COMBINED Signals.

nals results in error-free data after combination, demodulation and detection. Using selection combining with four signals, some errors remain but the bit error rate is still much lower by using all four signals.

\section{H. Bit-Error-Rate Characteristics}

The bit-error-rate characteristics can be calculated based on the received signal-to-noise ratios. For these calculations only measurement data recorded along the sideways track were used. In this measurement series the path loss is nearly constant. Inevitably some shadowing will be present, making the signal worse than Rayleigh distributed.

To be able to calculate relevant bit error rates, a new measurement series was recorded along the sideways track. The transmitted power was increased to $4 \mathrm{~mW}$ and the number of measurements was incremented to 300 data bursts, received along the sideways track. The other conditions for the measurement are comparable to the measurements discussed earlier in this text (section III-B, case 4).

Assuming the channel invariant during the time of one received burst, the bit error rates have been calculated for each measurement point, i.e. for each symbol burst, as

$$
P_{b}(i)=\frac{1}{2} \operatorname{erfc}\left(\sqrt{S N R_{D}(i)}\right)
$$

where $S N R_{D}(i)$ denotes the ratio of useful signal power to noise power at the detector during the i-th burst. The quantities $S N R_{D}(i)$ are computed from the one-sided noise power spectral density $N_{0}$ at the receiver, the measured squared channel gains $\left|h_{1}(i)\right|^{2}$ to $\left|h_{4}(i)\right|^{2}$ related to the different antennas/polarizations, and the considered signal combining strategy.

Calculating $P_{b}$ for all measurement points and averaging over the $N$ transmitted bursts yields $P_{b 1}$ :

$$
P_{b 1}=\frac{1}{2 N} \sum_{i=1}^{N} \operatorname{erfc}\left(\sqrt{S N R_{D}(i)}\right)
$$

This bit error rate is expressed as a function of the average $E_{b} / N_{0}$ per receive antenna, where $E_{b}$ denotes the average received bit energy per antenna. Assuming a QPSK constellation with variance equal to $1, E_{b}$ is computed as

$$
E_{b}=\frac{1}{8 N} \sum_{i=1}^{N}\left(\left|h_{1}(i)\right|^{2}+\left|h_{2}(i)\right|^{2}+\left|h_{3}(i)\right|^{2}+\left|h_{4}(i)\right|^{2}\right)
$$

The above $E_{b}$ corresponds to the average energy per bit received by the "average antenna", and results from the concatenation of all measurement series with the two dualpolarized antennas, involving all received signals, from all inputs. Thus, it represents the energy per bit averaged over the transmitted bursts and over the receiver branches, before combining. This method was adopted because the average signal level is different for each of the four antennas, which implies that selecting the $E_{b} / N_{0}$ of only one of the antennas would not be appropriate.

Based on the measured set of signal levels, the BER for different average $E_{b} / N_{0}$ values is calculated by scaling the recorded $S N R_{D}(i)$ and $E_{b}$ values by a common factor, followed by repeating (2), (3) and (4). The BER values obtained correspond to signals with the same distribution but different arithmetic means.

Fig. 15 shows the average BER as a function of the average $E_{b} / N_{0}$. The curve "average antenna" refers to the BER for a single antenna, averaged over the four antennas.

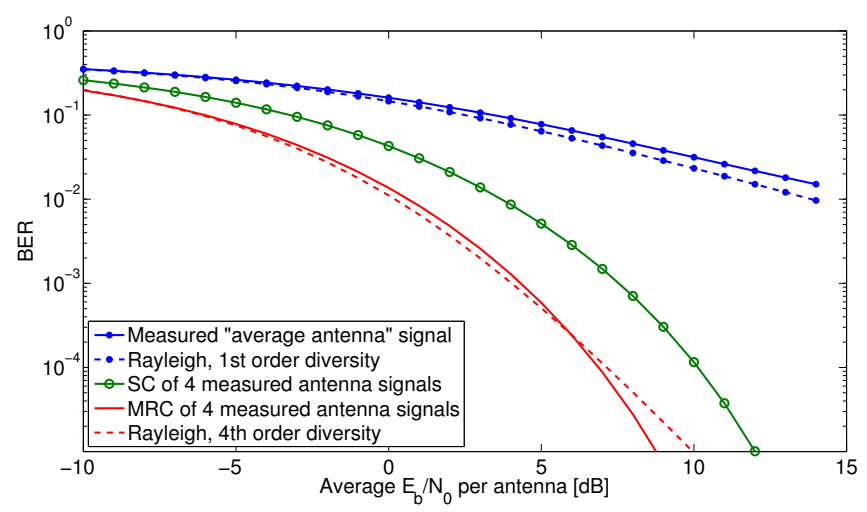

Fig. 15. BER characteristics as a function of average $E_{b} / N_{0}$ for the "average antenna". Both curves for selection combining (SC) and maximal ratio combining (MRC) of four antenna signals, result in a diversity order of four.

The four original antenna signals are combined using SC and MRC. The resulting graph in Fig. 15 shows array gain and diversity gain combined. The largest possible array gain for four antennas is $6 \mathrm{~dB}$, occuring when all four average signal strengths are equal.

Also shown in Fig. 15 are the theoretical BER curves for a single antenna and for MRC using four antennas, under the assumption of Rayleigh fading. The theoretical curves, displayed for $1^{\text {st }}$ - and $4^{\text {th }}$-order diversity, are calculated as $[15$, p. 825].

$$
P_{b 3}=\left[\frac{1}{2}(1-\mu)\right]^{L} \cdot \sum_{k=0}^{L-1}\left(\begin{array}{c}
L-1+k \\
k
\end{array}\right)\left[\frac{1}{2}(1+\mu)\right]^{k}
$$

with $L$ the diversity order and

$$
\mu=\sqrt{\frac{E_{b} / N_{0}}{1+E_{b} / N_{0}}}
$$

The curve for the average antenna reveals a higher bit error rate compared to the Rayleigh distributed signal. This is probably due to shadowing, both by the indoor environment 
and by the human body, resulting in a worse than Rayleigh distributed signal.

The bit error rates for the combined signals show a significant improvement, which is clearly best for MRC. For bit error rates down to $10^{-4}$, the curve for MRC approximates the theoretical characteristic for $4^{\text {th }}$-order diversity in a Rayleigh fading environment. Since the bit error rate for the original signals is a little higher than for Rayleigh fading, consequently, the bit error rate for the MRC combined signal is also slightly higher compared to the theoretical curve for Rayleigh fading with $4^{\text {th }}$-order diversity.

For bit error rates lower than $10^{-4}$ the curve for the measured signals deviates increasingly from the theoretical characteristic. This can be attributed to the absence of a sufficient number of very low signal levels after maximal ratio combining, resulting in an excessively low bit error rate. More measurements are needed to obtain more accurate values for the low error probabilities involved.

\section{CONCLUSIONS}

The measurement data clearly indicate the advantage of using two dual-polarized textile patch antennas for off-body communication.

The correlation values show that the signal levels for both feeds coming from the same patch antenna and corresponding to orthogonal polarizations fluctuate independently in our indoor test environment. Examining the data after demodulation and detection confirmed that the number of bit errors left after combining is lower than what would be obtained for each polarization alone. Hence the dual-polarized antenna, which produces two signals which are then MRC combined, provides a more reliable communication link compared to an antenna producing only one signal.

Using two dual-polarized antennas, on the front and rear of the body, is recommended, as the body always shields the radiation from the antenna such that no omnidirectional coverage around the body is possible with a single antenna. Using two antennas permits each antenna to cover a hemisphere around the body.

The pattern and polarization diversity complement each other to achieve a significant additional performance gain. The bit error rates and channel estimates after combining clearly demonstrate the performance increase by using all four signals. The bit-error-rate curves prove that an effective $4^{\text {th }}$-order diversity is realized in our application: the performance of the combined signal approximates the theoretical characteristics for $4^{\text {th }}$-order diversity in a Rayleigh fading environment when using MRC. Selection combining results in diversity of the same order but performs down to $2 \mathrm{~dB}$ worse compared to MRC.

The proposed antenna system is therefore a suitable candidate for future use in wearable textile systems for firefighters. The integrated research project "PROeTEX" [16] already developed some initial prototypes of the discussed wearable textile system including textile antennas and implementing diversity. Though, in the current prototypes, the antennas are fed by a non-textile portable transceiver, available on the market, implementing dual diversity [17]. The connection with the antenna terminals is realized with flexible coaxial cables [2]. Future research will aim to develop transceivers, connection cables and in general electronics, designed on or even completely made out of textile materials and therefore fully integrable into garments.

\section{REFERENCES}

[1] L. Van Langenhove, Smart textiles for medicine and healtcare. Cambridge, England: Woodhead Publishing Limited, 2007.

[2] D. Curone, G. Dudnik, G. Loriga, J. Luprano, G. Magenes, R. Paradiso, A. Tognetti, and A. Bonfiglio, "Smart Garments for Safety Improvement of Emergency/Disaster Operators," in Proc. 29th Annual Int. Conf. IEEE EMBS, 2007, pp. 3962-3965.

[3] P. S. Hall and Y. Hao, Antennas and Propagation for Body-Centric Wireless Communications. Boston/London: Artech House, 2006.

[4] C. Hertleer, H. Rogier, L. Vallozzi, and L. Van Langenhove, "A Textile Antenna for Off-Body Communication Integrated Into Protective Clothing for Firefighters," IEEE Trans. Antennas Propag., vol. 57, no. 4, pp. 919-925, Apr. 2009.

[5] T. F. Kennedy, P. W. Fink, A. W. Chu, N. J. Champagne, G. Y. Lin, and M. A. Khayat, "Body-Worn E-Textile Antennas: The Good, the LowMass, and the Conformal," IEEE Trans. Antennas Propag., vol. 57, no. 4, pp. 910-918, Apr. 2009.

[6] Y. Rahmat-Samii, "Wearable and Implantable Antennas in Body-Centric Communications," in Proc. 2nd European Conf. Antennas and Propag., EuCAP 2007, Edinburgh, UK, 2007, pp. 1-5.

[7] I. Khan, P. S. Hall, A. A. Serra, A. R. Guraliuc, and P. Nepa, "Diversity Performance Analysis for On-Body Communication Channels at 2.45 GHz," IEEE Trans. Antennas Propag., vol. 57, no. 4, pp. 956-963, Apr. 2009.

[8] I. Khan and P. S. Hall, "Multiple Antenna Reception at 5.8 and 10 GHz for Body-Centric Wireless Communication Channels," IEEE Trans. Antennas Propag., vol. 57, no. 1, pp. 248-255, Jan. 2009.

[9] Y. Ouyang, D. J. Love, and W. J. Chappell, "Body-Worn Distributed MIMO System," IEEE Trans. Vehicular Technology, vol. 58, no. 4, pp. 1752-1765, May 2009.

[10] S. L. Cotton and W. G. Scanlon, "Spatial Diversity and Correlation for Off-Body Communications in Indoor Environments at $868 \mathrm{MHz}$," in Proc. IEEE 65th Vehicular Technology Conf., VTC2007-Spring, Dublin, Ireland, 2007, pp. 372-376.

[11] C. Hertleer, A. Tronquo, H. Rogier, and L. Van Langenhove, "The use of textile materials to design wearable microstrip patch antennas," Textile Research Journal, vol. 78, no. 8, pp. 651-658, Aug. 2008.

[12] R. G. Vaughan, "Polarization Diversity in Mobile Communications," IEEE Trans. Vehicular Technology, vol. 39, no. 3, pp. 177-186, Aug. 1990.

[13] R. U. Nabar, H. Bölcskei, V. Erceg, D. Gesbert, and A. J. Paulraj, "Performance of Multiantenna Signaling Techniques in the Presence of Polarization Diversity," IEEE Trans. on Signal Processing, vol. 50, no. 10, pp. 2553-2562, Oct. 2002.

[14] L. Vallozzi, H. Rogier, and C. Hertleer, "Dual Polarized Textile Patch Antenna for Integration into Protective Garments," IEEE Antennas Wireless Prop. Lett., vol. 7, pp. 440-443, 2008.

[15] J. G. Proakis, Digital Communication. New York: McGraw-Hill, 2001.

[16] PROeTEX website: http://www.proetex.org/index.htm.

[17] "WLAN Reference Design with the MAX2830," Maxim Integrated Products. [Online]. Available: http://www.maxim-ic.com/appnotes.cfm/ an_pk/4276 


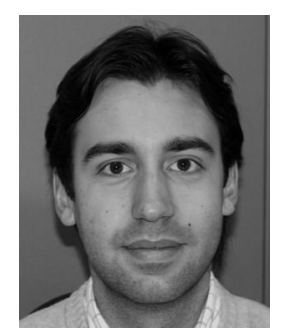

Luigi Vallozzi was born in Ortona, Italy, in 1980. $\mathrm{He}$ received the Laurea degree in electronic engineering from the Universit Politecnica delle Marche, Ancona, Italy, in 2005 and is currently pursuing the $\mathrm{Ph} . \mathrm{D}$. degree in electrical engineering at Ghent University, Ghent, Belgium. His research focuses on design and prototyping of antennas for wearable textile systems, and the modeling and characterization of multiple-input multiple-output wireless communication systems.

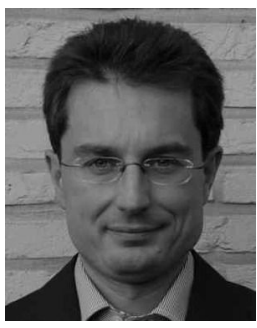

Patrick Van Torre was born in 1971. In 1995 he received the master's degree in Electrical Engineering from Hogeschool Gent, Belgium. From August 1995 till October 1998 he was working as development engineer in the private sector. Since November 1998 he has been active as educator in electronics and researcher in the field of ultrasound technology. He is currently employed by Hogeschool Gent where he teaches theory courses in Analog Electronics, organizes project oriented lab sessions and is involved in public relations activities and hardware development projects for third parties. He is part-time researcher, affiliated with the Department of Information Technology at Ghent University.

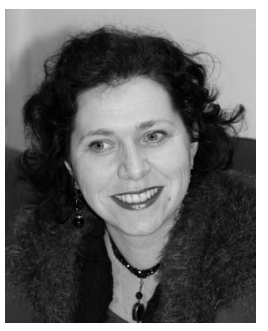

Carla Hertleer received the M. Sc. degree in textile engineering from Ghent University, Ghent, Belgium in 1990. For three years, she worked in a vertically integrated textile company that produces terry cloth. She worked during 6 years in a bank office but in June 2000, she decided to return to her roots: textiles. Since then, she works as a researcher at the Textile Department of Ghent University. She has given classes in weaving and Jacquard technology, but her recent activities are mainly concentrated on smart textiles, more specifically the research and development of textile sensors for integration in biomedical clothing and textile antennas. The latter research is carried out in collaboration with the Dept. of Information Technology of Ghent University. Her research is carried out in the framework of national and European projects. In 2009 she received her $\mathrm{PhD}$ in Engineering at Ghent University, on the research topic of textilebased antennas.

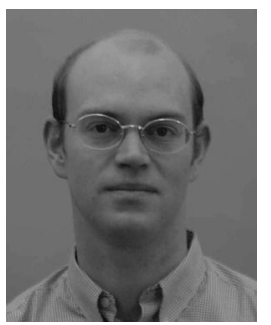

Hendrik Rogier was born in 1971. He received the Electrical Engineering and the Ph.D. degrees from Ghent University, Gent, Belgium, in 1994 and in 1999, respectively. He is currently a Postdoctoral Research Fellow of the Fund for Scientific Research Flanders (FWO-V), Department of Information Technology, Ghent University where he is also Associate Professor with the Department of Information Technology. From October 2003 to April 2004, he was a Visiting Scientist at the Mobile Communications Group of Vienna University of Technology. $\mathrm{He}$ authored and coauthored about 50 papers in international journals and about 70 contributions in conference proceedings. He is serving as a member of the Editorial Boarding of IET Science, Measurement Technology and acts as the URSI Commission B representative for Belgium. His current research interests are the analysis of electromagnetic waveguides, electromagnetic simulation techniques applied to electromagnetic compatibility (EMC) and signal integrity (SI) problems, as well as to indoor propagation and antenna design, and in smart antenna systems for wireless networks. Dr. Rogier was twice awarded the URSI Young Scientist Award, at the 2001 URSI Symposium on Electromagnetic Theory and at the 2002 URSI General Assembly. He is a Senior Member of the IEEE.

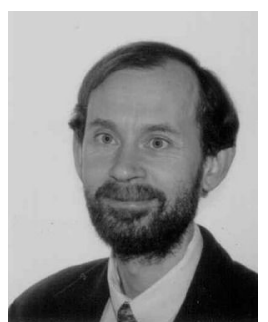

Marc Moeneclaey received the diploma of electrical engineering and the Ph.D. degree in electrical engineering from Ghent University, Gent, Belgium, in 1978 and 1983, respectively.

He is Professor at the Department of Telecommunications and Information Processing (TELIN), Gent University. His main research interests are in statistical communication theory, (iterative) estimation an detection, carrier and symbol synchronization, bandwidth-efficient modulation and coding, spreadspectrum, satellite and mobile communication. $\mathrm{He}$ is the author of more than 400 scientific papers in international journals and conference proceedings. Together with Prof. H. Meyr (RWTH Aachen) and Dr. S. Fechtel (Siemens AG), he co-authors the book Digital communication receivers - Synchronization, channel estimation, and signal processing. (J. Wiley, 1998). He is co-recipient of the Mannesmann Innovations Prize 2000. Since 2002, he has been a Fellow of IEEE.

During the period 1992-1994, was Editor for Synchronization, for the IEEE Transactions on Communications. He served as co-guest editor for special issues of the Wireless Personal Communications Journal (on Equalization and Synchronization in Wireless Communications) and the IEEE Journal on Selected Areas in Communications (on Signal Synchronization in Digital Transmission Systems) in 1998 and 2001, respectively.

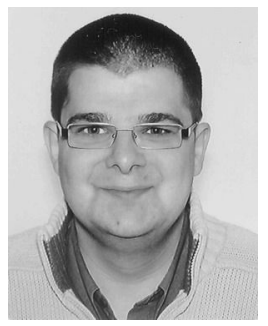

Jo Verhaevert received the engineering degree and the Ph.D. degree in electronic engineering from the Katholieke Universiteit Leuven, Belgium in 1999 and 2005, respectively. He currently teaches courses on telecommunication at the University College Ghent, department of Applied Engineering Sciences, Ghent, Belgium where he also performs research. His research interests include indoor wireless applications (such as wireless sensor networks), indoor propagation mechanisms and smart antenna systems for wireless systems. 